\title{
Fractional anisotropy of the fornix and hippocampal atrophy in Alzheimer's disease
}

\author{
Kejal Kantarci * \\ Department of Radiology, Mayo Clinic, Rochester, MN, USA
}

Edited by:

Kenichi Oishi, Johns Hopkins

University, USA

\section{Reviewed by:}

Roberta Brinton, University of

Southern California, USA

Hui Wang, Children's National Medical

Center, USA

\section{${ }^{*}$ Correspondence:}

Kejal Kantarci, Department of Radiology, Mayo Clinic, 200 First

Street SW, Rochester, MN 55905, USA

e-mail: kantarci.kejal@mayo.edu
Decrease in the directionality of water diffusion measured with fractional anisotropy (FA) on diffusion tensor imaging has been linked to loss of myelin and axons in the white matter. Fornix FA is consistently decreased in patients with mild cognitive impairment (MCl) and Alzheimer's disease (AD). Furthermore, decreased fornix FA is one of the earliest MRI abnormalities observed in cognitively normal individuals who are at an increased risk for $A D$, such as in pre-symptomatic carriers of familial $A D$ mutations and in pre-clinical $A D$. Reductions of FA at these early stages, which predicted the decline in memory function. Fornix carries the efferent projections from the CA1 and CA3 pyramidal neurons of the hippocampus and subiculum, connecting these structures to the septal nuclei, anterior thalamic nucleus, mammillary bodies, and medial hypothalamus. Fornix also carries the afferent cholinergic and GABAergic projections from the medial septal nuclei and the adjacent diagonal band back to the medial temporal lobe, interconnecting the core limbic structures. Because fornix carries the axons projecting from the hippocampus, integrity of the fornix is in-part linked to the integrity of the hippocampus. In keeping with that, fornix FA is reduced in subjects with hippocampal atrophy, correlating with memory function. The literature on FA reductions in the fornix in the clinical spectrum of $A D$ from pre-symptomatic carriers of familial $A D$ mutations to pre-clinical $A D, M C l$, and dementia stages is reviewed.

Keywords: fornix, DTI, Alzheimer's disease, hippocampus, MRI imaging

\section{ANATOMY OF THE HIPPOCAMPUS AND FORNIX CONNECTIONS}

Hippocampal projections to the subcortical structures that form the fimbria are carried by the fornix bundle. The fornix has two major components that connect hippocampus and subcortical structures. These are the larger postcommissural component and the smaller precommissural component. A majority of the axonal projections in the fornix originate from the subiculum of the hippocampus and form the postcommissural fornix carrying subicular axons to the mammillary bodies, which then project to the anterior nucleus of the thalamus. A major axonal tract originating from the anterior thalamus projects to the posterior cingulate cortex, which connects back to hippocampus via the cingulum tract closing the "Papez Circuit" (Benarroch, 2006). In addition, the precommissural fornix caries axons originating from the CA1 and CA3 pyramidal neurons of the hippocampus to the lateral septal nucleus, which projects to the medial septum. The medial septum and the nucleus of the diagonal band send cholinergic and GABAergic projections back to the medial temporal lobe via the fornix.

Damage to fornix in monkeys produces deficits in learning about the places of objects and about the places where responses should be made. They are impaired in using information about their place in an environment. Furthermore, fornix lesions impair conditional left-right discrimination learning based on visual appearance of an object on either side (Rolls, 2014). Fornix transection in human beings has been reported to produce significant and persistent anterograde amnesia (D'Esposito et al., 1995).
Because majority of the axonal projections carried by the fornix originate from the subiculum and to a lesser extent from CA1 and CA3 pyramidal neurons of the hippocampus, neurodegenerative processes impacting these neuronal populations would also lead to degeneration of the axonal projections originating from these neurons. However, it is important to note that not all axons in the fornix originate from the hippocampus, as the cholinergic and GABAergic connections originating from the medial septum and the nucleus of the diagonal band project back to the medial temporal lobe via the fornix. Therefore, degeneration of neuronal populations in the hippocampus may not fully explain the axonal loss in the fornix.

\section{NORMAL AGING AND THE HIPPOCAMPUS-FORNIX AXIS}

In monkeys, number of myelinated nerve fibers in the fornix decrease with aging by $15-34 \%$, but the loss of myelinated nerve fibers in the fornix is not disproportionately high or low compared to the rest of the white matter. However, it is not clear whether the myelinated nerve fiber loss from the fornix with age is due to loss of the parent neurons from the hippocampus, or due to age-related alterations that affect myelin sheaths, and alter the conduction velocities along nerve fibers resulting in degeneration in long projecting myelinated nerve fiber tracts. This loss of nerve fibers lead to a reduced subcortical connectivity of the hippocampus and may in-part be responsible for cognitive decline associated with normal aging even when the hippocampal neurons are intact (Peters et al., 2010).

Aging is associated with alterations in hippocampal dendritic morphology in the absence of neuronal loss, therefore 
age-associated structural alterations in the fornix less likely to originate from hippocampal neuronal loss, and more likely be the consequence of demyelination and degeneration of fornix fibers with age (Hof and Morrison, 2004). While both hippocampal volumes (Jack et al., 1998), and white matter fractional anisotropy (FA) decreases during aging (Walhovd et al., 2010), a relationship between fornix FA and hippocampal volume remains even after controlling for age. Using a recursive regression procedure, to evaluate sequential relationships between the alterations of the hippocampus and fornix, Pelletier et al. showed that hippocampal atrophy in healthy aging could be mediated by a loss of fornix connections potentially through a retrograde process (Pelletier et al., 2013). Longitudinal change in hippocampal volumes and fornix FA over the adult lifespan may be useful in determining whether hippocampal volume loss or a decrease in fornix FA occur earlier during the aging process.

Sex differences in structural network connectivity across the life span have been observed in DTI studies, with women showing greater overall cortical connectivity and network organization and greater efficiency than men (Gong et al., 2009). However, it is unclear at this time whether there is a difference in structural connectivity across the hippocampus-fornix axis among men and women.

\section{HIPPOCAMPUS-FORNIX AXIS IN PRODROMAL AND PRE-CLINICAL AD}

There is converging evidence from longitudinal studies on clinically followed and autopsied cohorts that most people with the amnestic form of mild cognitive impairment (MCI) who progress to dementia in the future, develop Alzheimer's disease (AD) (Flicker et al., 1991; Bowen et al., 1997; Morris et al., 2001; Bennett et al., 2002; Meyer et al., 2002; Jicha et al., 2006; Petersen et al., 2006). In keeping with these findings, both hippocampal atrophy and a reduction in fornix FA are frequently observed in amnestic MCI (Liu et al., 2011; Zhuang et al., 2012, 2013; Zhang et al., 2013) and are associated with memory decline (Mielke et al., 2012) and the risk of progression to AD (Mielke et al., 2012; Douaud et al., 2013).

The relationship between the structural integrity of hippocampus and fornix has been demonstrated in MCI (Mielke et al., 2012; Zhuang et al., 2012). In a cohort of cognitively normal (CN) older adults $(n=570$; median age $=78$; interquartile range $=74-83$ ) and MCI $(n=131$; median age $=80$; interquartile range $=77-$ 86) from the community, both $\mathrm{CN}$ and MCI patients showed decreased fornix FA if they also had hippocampal atrophy or AD pattern of hypometabolism on ${ }^{18}$ F Fluorodeoxyglucose (FDG) PET compared to the CN group with normal hippocampal volumes and metabolism on FDG PET. Having only a positive amyloid- $\beta$ PET scan with Pittsburgh compound-B (PiB) was not responsible for a reduction in fornix FA in $\mathrm{CN}$ individuals, demonstrating that high amyloid load does not influence diffusion tensor imaging (DTI)-based measures of white matter integrity in the absence of co-existent gray matter neurodegeneration in nondemented older adults (Figure 1) (Kantarci et al., 2014). Although individuals with $\mathrm{MCI}$ and pre-clinical $\mathrm{AD}$ have reductions in fornix FA, it should be noted that a reduction in fornix FA by itself would be insufficient in classifying preclinical and prodromal AD. However, fornix FA can be combined with other imaging

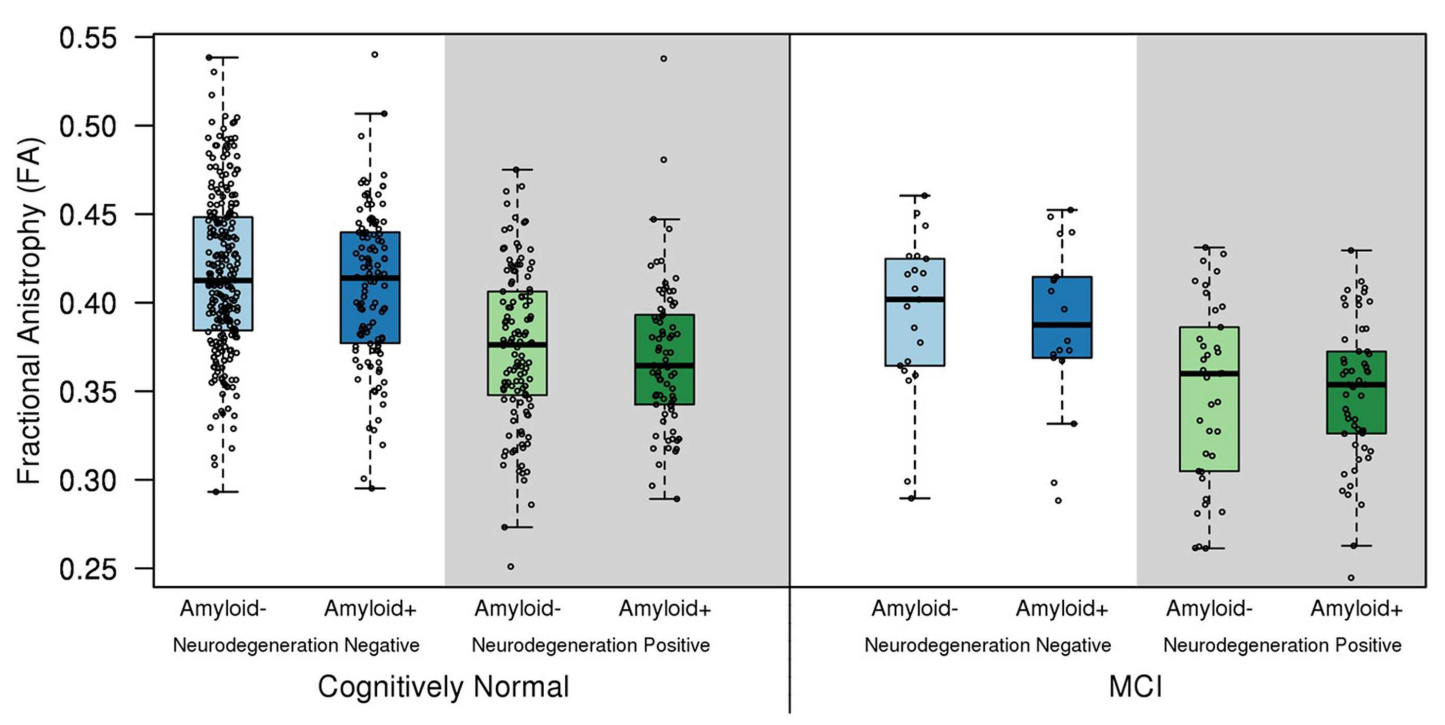

FIGURE 1 | Fractional anisotropy of the fornix in the cognitively normal (CN) and mild cognitive impairment (MCI) biomarker groups. Hippocampal atrophy on MRI and/or hypometabolism in the Alzheimer signature composite on FDG PET was used to classify subjects into the neurodegeneration-positive group, and high amyloid load on PET was used to classify subjects into the amyloid-positive group. Cut-points for amyloid positivity, hippocampal atrophy, and Alzheimer signature hypometabolism were determined from the 10th percentile of the measurement distributions in clinically diagnosed AD patients as previously described (Jack et al., 2012). Fornix FA was lower in the neurodegeneration positive $\mathrm{CN}$ and $\mathrm{MCl}$ patients compared to the cognitively normal group with normal imaging findings (amyloid and neurodegeneration negative; $p<0.001$ ). A positive amyloid PET scan was not associated with a reduction in fornix FA, in the absence of co-existent neurodegeneration in cognitively normal individuals $(p=0.19)$ (Kantarci et al., 2014). 
biomarkers such as hypometabolism on FDG PET, hippocampal atrophy, and amyloid- $\beta$ PET for a more accurate classification of individuals at risk for $\mathrm{AD}$, and predicting outcomes.

In carriers of the fully penetrant familial $\mathrm{AD}$ mutations (Ringman et al., 2007), a reduction in fornix FA was present in $\mathrm{CN}$ individuals who were destined to develop $\mathrm{AD}$ regardless of the cross-sectional area of the fornix, suggesting that the FA reduction in the fornix may precede hippocampal atrophy and clinical symptoms in $\mathrm{AD}$. It is not possible to make inferences on whether FA reductions precede hippocampal atrophy on MRI in the course of $\mathrm{AD}$ based on findings from cross-sectional studies. Longitudinal investigations with serial DTI and structural MRI in pre-clinical $\mathrm{AD}$ and MCI may clarify the sequence of DTI and volumetric MRI findings in $\mathrm{AD}$.

\section{HIPPOCAMPUS-FORNIX AXIS IN ALZHEIMER'S DISEASE}

There is a topographic concordance between gray and white matter diffusivity changes in AD (Kantarci et al., 2010), which implies that the disruption in white matter tracts is closely associated with the cortical pathology. Late-myelinating fibers in the brain such as the corticocortical association and the limbic pathways that are more vulnerable to degeneration related to $\mathrm{AD}$ than the earlymyelinating fibers such as the corticospinal tracts, which do not show any DTI abnormalities in early AD (Stricker et al., 2009; Kantarci et al., 2010; Oishi et al., 2012).

Consistent with the findings in normal aging and MCI and pre-clinical AD, correlations between fornix FA and hippocampal atrophy has been observed in AD (Firbank et al., 2007; Pievani et al., 2010; Lee et al., 2012). In a sub-regional analysis of the hippocampus Lee et al. reported that the strongest associations between the structural changes in the hippocampus and fornix diffusivity is localized to the hippocampal CA1 and anteromedial subiculum (Lee et al., 2012). CA1 and subiculum are the subregions of the hippocampus that are vulnerable to $\mathrm{AD}$-related neurodegeneration (Davies et al., 1992). Hippocampal efferent fibers that pass through the fornix are thought to originate from both of these subfields. The authors concluded that: "Cortical neuronal damage and subcortical axonal defects in $\mathrm{AD}$ are likely to be closely linked with each other, possibly reflecting a suggested pathogenic interaction between the two" (Lee et al., 2012). However, they also acknowledged that this hypothesis needs to be tested through longitudinal studies.

\section{CONCLUSION}

The association of microstructural alterations in the hippocampus and fornix continues throughout human lifespan and remains even when these structures are impacted by $\mathrm{AD}$ starting from the preclinical and prodromal stages. Prospective studies investigating the rates of structural alterations in these two structures may clarify the temporal sequence of the reductions in fornix FA and hippocampal volumes through preclinical, prodromal, and dementia stages of $\mathrm{AD}$.

\section{ACKNOWLEDGMENTS}

Dr. Kantarci's research program is supported by the R01 AG40042, P50 AG16574/Project1, and P50 AG44170/Project 2

\section{REFERENCES}

Benarroch, E. (2006). Basic Neurosciences with Clinical Applications. Philadelphia, PA: Elsevier.

Bennett, D. A., Wilson, R. S., Schneider, J. A., Evans, D. A., Beckett, L. A., Aggarwal, N. T., et al. (2002). Natural history of mild cognitive impairment in older persons. Neurology 59, 198-205. doi:10.1212/WNL.59.2.198

Bowen, J., Teri, L., Kukull, W., McCormick, W., McCurry, S. M., and Larson, E. B. (1997). Progression to dementia in patients with isolated memory loss. Lancet 349, 763-765. doi:10.1016/S0140-6736(96)08256-6

Davies, D. C., Horwood, N., Isaacs, S. L., and Mann, D. M. (1992). The effect of age and Alzheimer's disease on pyramidal neuron density in the individual fields of the hippocampal formation. Acta Neuropathol. 83, 510-517. doi:10.1007/BF00310028

D’Esposito, M., Verfaellie, M., Alexander, M. P., and Katz, D. I. (1995). Amnesia following traumatic bilateral fornix transection. Neurology 45, 1546-1550. doi:10.1212/WNL.45.8.1546

Douaud, G., Menke, R. A., Gass, A., Monsch, A. U., Rao, A., Whitcher, B., et al. (2013). Brain microstructure reveals early abnormalities more than two years prior to clinical progression from mild cognitive impairment to Alzheimer's disease. J. Neurosci. 33, 2147-2155. doi:10.1523/JNEUROSCI.4437-12.2013

Firbank, M. J., Blamire, A. M., Krishnan, M. S., Teodorczuk, A., English, P., Gholkar, A., et al. (2007). Diffusion tensor imaging in dementia with Lewy bodies and Alzheimer's disease. Psychiatry Res. 155, 135-145. doi:10.1016/j.pscychresns. 2007.01.001

Flicker, C., Ferris, S. H., and Reisberg, B. (1991). Mild cognitive impairment in the elderly: predictors of dementia. Neurology 41, 1006-1009. doi:10.1212/WNL.41. 7.1006

Gong, G., Rosa-Neto, P., Carbonell, F., Chen, Z. J., He, Y., and Evans, A. C. (2009). Age- and gender-related differences in the cortical anatomical network. J. Neurosci. 29, 15684-15693. doi:10.1523/JNEUROSCI.2308-09.2009

Hof, P. R., and Morrison, J. H. (2004). The aging brain: morphomolecular senescence of cortical circuits. Trends Neurosci. 27, 607-613. doi:10.1016/j.tins. 2004.07.013

Jack, C. R. Jr., Knopman, D. S., Weigand, S. D., Wiste, H. J., Vemuri, P., Lowe, V., et al. (2012). An operational approach to National Institute on Aging-Alzheimer's Association criteria for preclinical Alzheimer disease. Ann. Neurol. 71, 765-775. doi:10.1002/ana.22628

Jack, C. R. Jr., Petersen, R. C., Xu, Y., O’Brien, P. C., Smith, G. E., Ivnik, R. J., et al. (1998). Rate of medial temporal lobe atrophy in typical aging and Alzheimer's disease. Neurology 51, 993-999.

Jicha, G. A., Parisi, J. E., Dickson, D. W., Johnson, K., Cha, R., Ivnik, R. J., et al. (2006). Neuropathologic outcome of mild cognitive impairment following progression to clinical dementia. Arch Neurol 63, 674-681. doi:10.1001/archneur.63.5.674

Kantarci, K., Avula, R., Senjem, M. L., Samikoglu, A. R., Zhang, B., Weigand, S. D., et al. (2010). Dementia with Lewy bodies and Alzheimer disease: neurodegenerative patterns characterized by DTI. Neurology 74, 1814-1821. doi:10.1212/ WNL.0b013e3181e0f7cf

Kantarci, K., Schwarz, C. G., Reid, R. I., Przybelski, S. A., Lesnick, T. G., Zuk, S. M., et al. (2014). White matter integrity on DTI, amyloid load, and neurodegeneration in non-demented elderly. JAMA Neurol. doi:10.1001/ jamaneurol.2014.1482

Lee, D. Y., Fletcher, E., Carmichael, O. T., Singh, B., Mungas, D., Reed, B., et al. (2012). Sub-regional hippocampal injury is associated with fornix degeneration in Alzheimer's disease. Front. Aging Neurosci. 4:1. doi:10.3389/fnagi.2012.00001

Liu, Y., Spulber, G., Lehtimaki, K. K., Kononen, M., Hallikainen, I., Grohn, H., et al. (2011). Diffusion tensor imaging and tract-based spatial statistics in Alzheimer's disease and mild cognitive impairment. Neurobiol. Aging. 32, 1558-1571. doi:10.1016/j.neurobiolaging.2009.10.006

Meyer, J. S., Xu, G., Thornby, J., Chowdhury, M. H., and Quach, M. (2002). Is mild cognitive impairment prodromal for vascular dementia like Alzheimer's disease? Stroke 33, 1981-1985. doi:10.1161/01.STR.0000024432.34557.10

Mielke, M. M., Okonkwo, O. C., Oishi, K., Mori, S., Tighe, S., Miller, M. I., et al. (2012). Fornix integrity and hippocampal volume predict memory decline and progression to Alzheimer's disease. Alzheimers Dement. 8, 105-113. doi:10.1016/ j.jalz.2011.05.2416

Morris, J. C., Storandt, M., Miller, J. P., McKeel, D. W., Price, J. L., Rubin, E. H., et al. (2001). Mild cognitive impairment represents early-stage Alzheimer disease. Arch. Neurol. 58, 397-405. doi:10.1001/archneur.58.3.397 
Oishi, K., Mielke, M. M., Albert, M., Lyketsos, C. G., and Mori, S. (2012). The fornix sign: a potential sign for Alzheimer's disease based on diffusion tensor imaging. J. Neuroimaging 22, 365-374. doi:10.1111/j.1552-6569.2011.00633.x

Pelletier, A., Periot, O., Dilharreguy, B., Hiba, B., Bordessoules, M., Peres, K., et al. (2013). Structural hippocampal network alterations during healthy aging: a multi-modal MRI study. Front. Aging Neurosci. 5:84. doi:10.3389/fnagi.2013. 00084

Peters, A., Sethares, C., and Moss, M. B. (2010). How the primate fornix is affected by age. J. Comp. Neurol. 518, 3962-3980. doi:10.1002/cne.22434

Petersen, R. C., Parisi, J. E., Dickson, D. W., Johnson, K. A., Knopman, D. S., Boeve, B. F., et al. (2006). Neuropathologic features of amnestic mild cognitive impairment. Arch. Neurol. 63, 665-672. doi:10.1001/archneur.63.5.665

Pievani, M., Agosta, F., Pagani, E., Canu, E., Sala, S., Absinta, M., et al. (2010). Assessment of white matter tract damage in mild cognitive impairment and Alzheimer's disease. Hum. Brain Mapp. 31, 1862-1875. doi:10.1002/hbm.20978

Ringman, J. M., O’Neill, J., Geschwind, D., Medina, L., Apostolova, L. G., Rodriguez, Y., et al. (2007). Diffusion tensor imaging in preclinical and presymptomatic carriers of familial Alzheimer's disease mutations. Brain 130, 1767-1776. doi:10.1093/brain/awm102

Rolls, E. T. (2014). Limbic systems for emotion and memory but no single limbic system. Cortex. doi:10.1016/j.cortex.2013.12.005

Stricker, N. H., Schweinsburg, B. C., Delano-Wood, L., Wierenga, C. E., Bangen, K. J., Haaland, K. Y., et al. (2009). Decreased white matter integrity in latemyelinating fiber pathways in Alzheimer's disease supports retrogenesis. Neuroimage 45, 10-16. doi:10.1016/j.neuroimage.2008.11.027

Walhovd, K. B., Westlye, L. T., Moe, V., Slinning, K., Due-Tonnessen, P., Bjornerud, A., et al. (2010). White matter characteristics and cognition in prenatally opiateand polysubstance-exposed children: a diffusion tensor imaging study. AJNR Am. J. Neuroradiol. 31, 894-900. doi:10.3174/ajnr.A1957
Zhang, Y., Schuff, N., Camacho, M., Chao, L. L., Fletcher, T. P., Yaffe, K., et al. (2013). MRI markers for mild cognitive impairment: comparisons between white matter integrity and gray matter volume measurements. PLoS ONE 8:e66367. doi:10.1371/journal.pone.0066367

Zhuang, L., Sachdev, P. S., Trollor, J. N., Reppermund, S., Kochan, N. A., Brodaty, H., et al. (2013). Microstructural white matter changes, not hippocampal atrophy, detect early amnestic mild cognitive impairment. PLoS ONE 8:e58887. doi:10.1371/journal.pone.0058887

Zhuang, L., Wen, W., Trollor, J. N., Kochan, N. A., Reppermund, S., Brodaty, H., et al. (2012). Abnormalities of the fornix in mild cognitive impairment are related to episodic memory loss. J Alzheimers Dis 29, 629-639. doi:10.3233/JAD-2012111766

Conflict of Interest Statement: The author declares that the research was conducted in the absence of any commercial or financial relationships that could be construed as a potential conflict of interest.

Received: 11 August 2014; paper pending published: 05 October 2014; accepted: 27 October 2014; published online: 13 November 2014.

Citation: Kantarci K (2014) Fractional anisotropy of the fornix and hippocampal atrophy in Alzheimer's disease. Front. Aging Neurosci. 6:316. doi: 10.3389/fnagi.2014.00316 This article was submitted to the journal Frontiers in Aging Neuroscience.

Copyright (C) 2014 Kantarci. This is an open-access article distributed under the terms of the Creative Commons Attribution License (CC BY). The use, distribution or reproduction in other forums is permitted, provided the original author(s) or licensor are credited and that the original publication in this journal is cited, in accordance with accepted academic practice. No use, distribution or reproduction is permitted which does not comply with these terms. 